\title{
ON THE EQUIVALENCE OF INTEGRAL REPRESENTATIONS OF GROUPS
}

\section{A. BIAEYNICKI-BIRULA}

ABstract. Let $R$ be a local ring and let $R^{\prime}$ be a commutative $R$-algebra faithfully flat as an $R$-module. Let $G$ be a finitely generated group and let $M, N$ be $R G$-modules, finitely presented over $R$. Let $M^{\prime}=M \otimes_{R} R^{\prime} ; N^{\prime}=N \otimes_{R} R^{\prime}$, then $M^{\prime}, N^{\prime}$ can be considered as $R^{\prime} G$-modules. We shall prove that the $R^{\prime} G$-modules $M^{\prime}, N^{\prime}$ are isomorphic if and only if the $R G$-modules $M$ and $N$ are isomorphic. The proof depends on a theorem on noncommutative cohomology which is presented in the first part of the paper.

1. All considered rings are assumed to have an identity 1 and all modules are supposed to be unitary. Subrings are assumed to have the same identity as the containing rings. For any ring $P$, let $P^{*}$ denote the group of all invertible elements of $P$. For any two $P$-modules $M$ and $N$ we shall write $M \approx_{P} N$ if $M$ is isomorphic to $N$.

In the paper we shall use some notions of Galois theory, Galois cohomology and Amitsur cohomology. We shall now recall briefly some basic definitions of these theories. Two automorphisms $g, h$ of a commutative ring $P$ are called strongly different if for every nonzero idempotent $e$ of $P$ there exists $s$ in $P$ such that $f(s) e \neq g(s) e$. If $G$ is a finite group of automorphisms of a commutative ring $P^{\prime}$, $P=\left(P^{\prime}\right)^{G}, P^{\prime}$ is a separable $P$-algebra and the elements of $G$ are pairwise strongly different, then $P^{\prime}$ is called a Galois extension of $P$ with Galois group $G$ (for details see [3]).

Assume now that $P^{\prime}$ is a Galois extension of $P$ with Galois group $G$ and let $A$ be a (in general noncommutative) $P$-algebra. Then $H^{1}\left(G,\left(A \otimes_{P} P^{\prime}\right)^{*}\right)$ denotes the first cohomology set of $G$ with coefficients in $\left(A \otimes P^{\prime}\right)^{*}$, where the action of $G$ on $\left(A \otimes P^{\prime}\right)^{*}$ is defined via the formula $g\left(a \otimes p^{\prime}\right)=a \otimes g\left(p^{\prime}\right)$, for any $a \in A, p^{\prime} \in P^{\prime}, g \in G$ (for definition see, e.g., [7, p. 131]).

On the other hand if $P$ is any commutative ring and $F$ any functor defined on the category of commutative $P$-algebras into the category of all groups then for any commutative $P$-algebra $P^{\prime}$ there exists the first Amitsur cohomology set $H^{1}\left(P^{\prime} / P, F\right)$ defined in the following way. Consider the following diagram

Received by the editors November 12, 1969 and, in revised form, March 10, 1970. AMS 1969 subject classifications. Primary 2080, 1640.

Key words and phrases. Algebras over local rings, representations of algebras, integral representations of groups, faithfully flat commutative algebras, noncommutative Amitsur cohomology. 


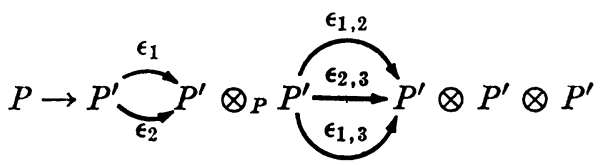

where $\quad \epsilon_{1}(a)=a \otimes 1, \quad \epsilon_{2}(a)=1 \otimes a, \quad \epsilon_{1,2}(a \otimes b)=a \otimes b \otimes 1, \quad \epsilon_{2,3}(a \otimes b)$ $=1 \otimes a \otimes b, \epsilon_{1,3}(a \otimes b)=a \otimes 1 \otimes b$, for any $a, b \in P^{\prime}$. Then $u \in F\left(P^{\prime} \otimes P^{\prime}\right)$ is said to be a cocycle if $\left[F\left(\epsilon_{1,2}\right)(u)\right] \cdot\left[F\left(\epsilon_{2,3}\right)(u)\right]=F\left(\epsilon_{1,3}\right)(u)$. The set of cocycles is denoted by $Z^{1}\left(P^{\prime} / P, F\right)$. Cocycles $u, v \in Z^{1}\left(P^{\prime} / P, F\right)$ are said to be cohomologous if there exists $t \in F\left(P^{\prime}\right)$ such that $v=\left[F\left(\epsilon_{1}\right) t\right] u\left[F\left(\epsilon_{2}\right) t\right]^{-1}$. This defines an equivalence relation on the set $Z^{1}\left(P^{\prime} / P, F\right)$, and $H^{1}\left(P^{\prime} / P, F\right)$ is defined as the set of equivalence classes of the relation.

Let $A$ be a (in general noncommutative) $P$-algebra and let $M, N$ be $A$-modules such that $M \otimes P^{\prime} \sim_{A \otimes P^{\prime}} N \otimes P^{\prime}$. Then we may choose an isomorphism $\tau: M \otimes P^{\prime} \rightarrow N \otimes P^{\prime}$. Let $\tau_{1}, \tau_{2}: M \otimes P^{\prime} \otimes P^{\prime} \rightarrow N \otimes P^{\prime} \otimes P^{\prime}$ be the isomorphisms induced by $\tau$ and $\epsilon_{1}, \epsilon_{2}$, respectively. Then $\tau_{2}^{-1} \tau_{1} \in \mathrm{Aut}_{A} \otimes P^{\prime} \otimes P^{\prime}\left(M \otimes P^{\prime} \otimes P^{\prime}\right)$ and it is easy to show that in fact $\tau_{2}^{-1} \tau_{1} \in Z^{1}\left(P^{\prime} / P\right.$, Aut $\left.\left(M \otimes_{P} \cdots\right)\right)$. One can also directly verify that the cohomology class of $\tau_{2}^{-1} \tau_{1}$ does not depend on the particular choice of $\tau$. Therefore there exists a map of the set of isomorphism classes of $A$-modules $N$ such that $M \otimes P^{\prime} \sim N \otimes P^{\prime}$ into $H^{1}\left(P^{\prime} / P, \operatorname{Aut}\left(M \otimes_{P} \cdots\right)\right)$ and one can prove that if $P^{\prime}$ is faithfully flat over $P, A$ is finitely generated (as an algebra over $P$ ) and $M$ is finitely presented as $P$-module then the map is injective. Indeed suppose that the above assumptions hold. It is easy to check that if two $A$-modules $N, N^{\prime}$ have the same image in $H^{1}\left(P^{\prime} / P, \operatorname{Aut}\left(M \otimes_{P} \cdots\right)\right)$ then there exists an isomorphism $\phi: N \otimes P^{\prime} \rightarrow N^{\prime} \otimes P^{\prime}$ such that the isomorphisms $\phi_{1}, \phi_{2}: N \otimes P^{\prime} \otimes P^{\prime}$ $\rightarrow N^{\prime} \otimes P^{\prime} \otimes P^{\prime}$, induced by $\phi$ and $\epsilon_{1}, \epsilon_{2}$, coincide. Hence it suffices to show that the diagram

$$
\begin{aligned}
0 & \rightarrow \operatorname{Hom}_{A}\left(N, N^{\prime}\right) \rightarrow \operatorname{Hom}_{A} \otimes P^{\prime}\left(N \otimes P^{\prime}, N^{\prime} \otimes P^{\prime}\right) \\
& \rightarrow \operatorname{Hom}_{A} \otimes P^{\prime} \otimes P^{\prime} \\
& \left(N \otimes P^{\prime} \otimes P^{\prime}, N^{\prime} \otimes P^{\prime} \otimes P^{\prime}\right),
\end{aligned}
$$

induced by the sequence

$$
0 \rightarrow P \rightarrow P^{\prime \underset{\epsilon_{2}}{\stackrel{\epsilon_{1}}{\rightarrow}}} P^{\prime} \otimes P^{\prime},
$$

is exact. This follows from Lemma 1.1, p. 18 of [5] and the following result (applied for $P_{1}=P^{\prime}$ and $P_{1}=P^{\prime} \otimes P^{\prime}$ ).

(A) Let $P$ be a commutative ring and let $A$ be a (in general noncommutative) $P$-algebra finitely generated over $P$ (as an algebra). 
Let $N, N^{\prime}$ be $A$-modules finitely presented as $P$-modules and let $P_{1}$ be any faithfully flat commutative $P$-algebra. Then the canonical homomorphism

$$
\operatorname{Hom}_{A}\left(N, N^{\prime}\right) \otimes P_{1} \rightarrow \operatorname{Hom}_{A \otimes P_{1}}\left(N \otimes P_{1}, N^{\prime} \otimes P_{1}\right)
$$

is an isomorphism.

In fact if $S$ is a finite set of generators of $A$ over $P$ then there is the following exact sequence

$$
0 \rightarrow \operatorname{Hom}_{A}\left(N, N^{\prime}\right) \hookrightarrow \operatorname{Hom}_{P}\left(N, N^{\prime}\right) \stackrel{\phi}{\rightarrow} \operatorname{Hom}_{P}\left(N, N^{\prime}\right)^{\mathbf{s}},
$$

where $\phi(h)=(s h-h s)_{s \in s}$. Since $P_{1}$ is faithfully flat over $P$, the sequence

$$
\begin{aligned}
0 & \rightarrow \operatorname{Hom}_{A}\left(N, N^{\prime}\right) \otimes P_{1} \\
& \hookrightarrow \operatorname{Hom}_{P}\left(N, N^{\prime}\right) \otimes P_{1} \stackrel{\phi \otimes 1}{\longrightarrow}\left(\operatorname{Hom}_{P}\left(N, N^{\prime}\right) \otimes P_{1}\right)^{S}
\end{aligned}
$$

is also exact and (A) follows from the fact that the canonical homomorphism $\operatorname{Hom}_{P}\left(N, N^{\prime}\right) \otimes P_{1} \rightarrow \operatorname{Hom}_{P_{1}}\left(N \otimes P_{1}, N^{\prime} \otimes P_{1}\right)$ is an isomorphism [1, Proposition 11, p. 39].

2. Let $R$ be a local ring and let $E$ be a (in general noncommutative) $R$-algebra with 1 . Let $R^{\prime}$ be a Galois extension of $R$ (in the sense of [3]) with Galois group $G$.

Lemma 1. Let $R, R^{\prime}, A$ be as above and let $H^{1}\left(R^{\prime} / R,(E \otimes \cdots)^{*}\right)$ be the first cohomology set of the noncommutative Amitsur cohomology then

$$
H^{1}\left(R^{\prime} / R,\left(E \otimes_{R} \cdots\right)^{*}\right)=H^{1}\left(G,\left(E \otimes R^{\prime}\right)^{*}\right)
$$

where the action of $G$ on $\left(E \otimes R^{\prime}\right)^{*}$ is defined via the formula $g\left(a \otimes r^{\prime}\right)$ $=a \otimes g\left(r^{\prime}\right)$, for any $a \in E, r^{\prime} \in R^{\prime}, g \in G$.

Proof. If $E$ is a commutative $R$-algebra then the result is a particular case of Theorem 5.4 of [3]. The argument in the general case is analogous.

Let $R$ be a local ring, $m$ its maximal ideal and let $E$ be an $R$-algebra. We say that $E$ is of type (*) if the $R / m$-algebra $F=E \otimes R / m$ is finite dimensional, and if $\phi$ is the canonical morphism $E \rightarrow F$ then for any $R$-algebra $R^{\prime}$ projective and of finite type as $R$-module, $a \in\left(E \otimes R^{\prime}\right)^{*}$ if and only if $(\phi \otimes 1)(a) \in\left(F \otimes{ }_{R} R^{\prime}\right)^{*}$.

Note that if $E$ is finitely generated as an $R$-module then $E$ is of type (*). In fact, let $F=E \otimes R / m$ and $\phi(a)=a \otimes 1$, for every $a \in E$. It sufficies to show that if $R^{\prime}$ is a faithfully flat $R$-algebra and 
$(\phi \otimes 1)(a) \in\left(E \otimes R / m \otimes R^{\prime}\right)^{*}$ for some $a \in E \otimes R^{\prime}$ then $a$ is invertible in $E \otimes R^{\prime}$. Consider the homomorphism $\phi_{a}: E \otimes R^{\prime} \rightarrow E \otimes R^{\prime}$ which sends every $x \in E \otimes R^{\prime}$ onto $a x$. $\phi_{a}$ induces an isomorphism $E \otimes R^{\prime} \otimes R / m \rightarrow E \otimes R^{\prime} \otimes R / m$. Since $E \otimes R^{\prime} \otimes R / m \approx\left(E \otimes R^{\prime}\right) \otimes_{R^{\prime}} R^{\prime} / m R^{\prime}$ and $m R^{\prime}$ is contained in the radical of $R^{\prime}$ hence by Corollaire 1 , p. 105 in [1], $\phi_{a}$ is an epimorphism. Therefore $a$ has a right-inverse. Similarly the element $a$ has a left-inverse. Thus $a \in\left(E \otimes R^{\prime}\right)^{*}$.

THEOREM 1. Let $R, R^{\prime}, E$ be as in Lemma 1 and suppose that $E$ is of type (*). Then $H^{1}\left(R^{\prime} / R,\left(E \otimes_{R} \cdots\right)^{*}\right)$ is trivial.

Proof. It follows from Lemma 1 that it suffices to show that $H^{1}\left(G,\left(E \otimes_{R} \cdots\right)^{*}\right)$ is trivial, where $G$ is any group of automorphisms of $R^{\prime} / R$ such that $R^{\prime}$ is a Galois extension of $R$ with Galois group $G$.

Step. 1. Assume that $R$ is a field, $E$ is a finite-dimensional $R$-algebra, and $R^{\prime}$ is a finite Galois field extension of $R$. In this case the result is well known (see Exercise 2, p. 160 in [7]).

Step 2. Assume that $R$ is a field, $E$ is a finite-dimensional $R$-algebra and $R^{\prime}$ any finite-dimensional $R$-algebra such that $R^{\prime}=\bigoplus_{i=1}^{r} R_{i}$, where $R_{i}=R_{j}$ for $i, j=1, \cdots, r$ and $R_{1}$ (and hence $R_{i}$ for $i=1, \cdots$, $r)$ is a finite Galois field extension of $R$. Let $1=e_{1}+\cdots+e_{r}$ be the corresponding decomposition of $1 \in R^{\prime}$ into a sum of orthogonal idempotents. Let $G$ be the direct product of the Galois group $H$ of $R_{1}$ over $R$ and of any group $G^{\prime}$ acting freely and transitively on the set $\{1, \cdots, r\}$. Then $G$ acts in a natural way on $R^{\prime}$, and $R^{\prime}$ is a Galois extension of $R$ with Galois group $G$. Let $\left\{T_{0}\right\}_{0 \in G}$ be a 1cocycle with values in $\left(E \otimes R^{\prime}\right)^{*}$ (i.e. $T_{g} \in\left(E \otimes R^{\prime}\right)^{*}, T_{g h}=g\left(T_{h}\right) \cdot T_{g}$, for any $g, h \in G)$. Then $\left\{T_{h} \cdot e_{1}\right\}_{h \in H}$ can be interpreted as a 1 -cocycle with values in $\left(E \otimes R_{1}\right)^{*}$ and hence it follows from the result of the first step that we may assume $T_{h} \cdot e_{1}=e_{1}$, for every $h \in H$. Let $a$ be any element of $R_{1}$ such that $\sum_{h \in H} h(a)=1$ and let $T=\sum_{o \in G} T_{o} \cdot g\left(a e_{1}\right)$. Then it is easy to see that $T \in\left(E \otimes R^{\prime}\right)^{*}$ and $g(T)=T_{0}^{-1} T$ for any $g \in G$. Hence the cocycle $\left\{T_{0}\right\}$ is coboundary of a 0 -cochain.

Step 3. The general case. Let $m$ be the maximal ideal of $R$. Then $R^{\prime} / m R^{\prime}\left(=R^{\prime} \otimes R / m\right)$ is a Galois extension of $R / m$ with Galois group $G$ (acting via the formula $g(a \otimes s)=g(a) \otimes s$, for any $a \in R^{\prime}, s \in R / m$, $g \in G)$ [3, Lemma 1.7]. Let $\left\{T_{o}\right\}_{o \in G}$ be a 1 -cocycle with values in $\left(E \otimes R^{\prime}\right)^{*}$ and let $\left\{\bar{T}_{\theta}\right\}_{\theta \in G}$ be the image under the homomorphism $\phi$ (defined as in the definition of algebras of type (*)). Then it follows from Step 2 that there exists $\bar{T} \in\left((\phi \otimes 1)\left(E \otimes R^{\prime}\right)\right)^{*}$ such that $\bar{T}_{\theta}=g\left(\bar{T}^{-1}\right) \bar{T}$ and hence $\sum_{g \in G} g(a \bar{T}) \bar{T}_{\theta}$ is an invertible element of $\left((\phi \otimes 1)\left(E \otimes R^{\prime}\right)\right)^{*}$, for some $a \in R^{\prime} / m R^{\prime}$ (it suffices to take any ele- 
ment $a \in R^{\prime} / m R^{\prime}$ such that $\sum_{g \in G} g(a)=1$; existence of such an element follows from Lemma 1.6 in [3]). Let $T$ be any element of $E \otimes R^{\prime}$ whose image under $\phi \otimes 1$ equals $a \bar{T}$. Then $T \in E \otimes R^{\prime}$ and $\sum_{g \in G} g(T) T_{g}$ $\in\left(E \otimes R^{\prime}\right)^{*}$ (since the image of the element $\sum_{g \in G} g(T) T_{g}$ under $\phi \otimes 1$ is invertible in $\phi\left(E \otimes R^{\prime}\right)$ and $E$ with $\phi$ satisfies conditions of the definition of algebras of type (*)). Let $T_{1}=\sum g(T) T_{0}$ then $g\left(T_{1}\right)$ $=T_{1} T_{g}^{-1}$, for any $g \in G$. Hence $\left\{T_{0}\right\}_{o \in G}$ is a coboundary and this completes the proof of the theorem.

3. TheOREM 2. Let $R$ be a local ring, $A$ a finilely generated $R$-algebra (e.g. $A=R G$, where $G$ is any finite group) and let $M, N$ be (left) $A$ modules finitely presented as modules over $R$. Let $R^{\prime}$ be a commutative $R$-algebra faithfully flat as $R$-module. Then $M \otimes R^{\prime} \approx_{A} \otimes_{R^{\prime}} N \otimes R^{\prime}$ if and only if $M \approx{ }_{A} N$.

First we shall prove the following lemma.

LEMMA 2. Let $R$ be a semilocal ring in which all residue fields contain more than $k$ elements. Let $A$ be a finitely generated $R$-algebra. Finally let $M, N$ be two $A$-modules finitely presented over $R$ and generated by sets composed of at most $k$ elements and let $R^{\prime}$ be a commutative $R$-algebra faithfully flat over $R$. Then $M \otimes R^{\prime} \approx{ }_{A} \otimes_{R}, N \times R^{\prime}$ implies $M \approx{ }_{A} N$.

Proof. Let $\tau: M \otimes R^{\prime} \rightarrow N \otimes R^{\prime}$ be an isomorphism. By (A) (see $\S 1), \operatorname{Hom}_{A} \otimes R^{\prime}\left(M \otimes R^{\prime}, \quad N \otimes R^{\prime}\right) \approx \operatorname{Hom}_{A}(M, N) \otimes R^{\prime}$. Hence $\tau=a_{1} \beta_{1}+\cdots+a_{r} \beta_{r}$, where $a_{1}, \cdots, a_{r} \in R^{\prime}, \beta_{1}, \cdots, \beta_{r} \in \operatorname{Hom}_{A}(M, N)$. Let $m_{1}, \cdots, m_{s}$ be all the maximal ideals of $R$. Let $\beta_{1, i}, \cdots, \beta_{r, i}$ be the induced homomorphisms of $R / m_{i}$-vector spaces $M \otimes R / m_{i}$ $\rightarrow N \otimes R / m_{i}$. Then as in the proof of the Noether-Deuring theorem (see e.g. [4]) we see that there exist $c_{1, i}^{\prime}, \cdots, c_{r, i}^{\prime} \in R / m_{i}$ such that $c_{1, i}^{\prime} \beta_{1, i}^{\prime}+\cdots+c_{r, i} \beta_{r, i}^{\prime}$ is an isomorphism of $M \otimes R / m_{i}$ onto $N \otimes R / m_{i}$ (since $R / m_{1}$ contains at least $k$ elements). Then we may choose elements $c_{1}, \cdots, c_{r} \in R$ such that $c_{j} \equiv c_{j, i} \bmod m_{i}$, for $j=1, \cdots, r$, $i=1, \cdots, s$. Therefore $\phi=c_{1} \beta_{1}+\cdots+c_{r} \beta_{r}$ induces an isomorphism $M \otimes R / m_{i} \rightarrow N \otimes R / m_{i}$ for $i=1, \cdots, s$. By [1, Proposition 11, p. 113], $\phi$ is surjective. Therefore $\phi \otimes 1: M \otimes R^{\prime} \rightarrow N \otimes R^{\prime}$ is surjective, but on the other hand $M \otimes R^{\prime} \approx N \otimes R^{\prime}$. Hence it follows from Proposi8.9.3 in [6] that $\phi$ is an isomorphism.

REMARK. Let $R$ be a semilocal ring with radical $m$ and let $R^{\prime}$ be a faithfully flat $R$-algebra such that $R / m \approx R^{\prime} / m R^{\prime}$. Suppose that $A$ is a finitely generated $R$-algebra and let $M, N$ be two $A$-modules finitely presented as $R$-modules. Then it follows from the proof of the lemma that $M \otimes R^{\prime} \approx{ }_{A} \otimes R^{\prime} N \otimes R^{\prime}$ implies $M \approx_{A} N$. Therefore if $m_{1}, \cdots, m_{\varepsilon}$ are all the maximal ideals and 


$$
M \otimes R_{m_{i}} \approx \Delta \otimes R_{m_{i}} N \otimes R_{m_{i}} \text { for } i=1, \cdots, s
$$

then $M \approx{ }_{\Lambda} N$. In fact the $R$-algebra $R^{\prime}=R_{m_{1}} \oplus \cdots \oplus R_{m_{s}}$ satisfies the above assumptions.

ProOF OF THEOREM 2. Of course if $M \approx_{A} N$ then $M \otimes_{R} R^{\prime}$ $\approx_{A} \otimes_{R^{\prime}} N \otimes_{R} R^{\prime}$. Hence we shall assume that $M \otimes_{R} R^{\prime} \approx_{A} \otimes_{R} R^{\prime} N \otimes_{R} R^{\prime}$. It follows from the above lemma that it suffices to consider the case where $R / m$ is finite (where $m$ is the maximal ideal of $R$ ). Let $n \geqq k$, let $f=x^{n}+a_{n-1} x^{n-1}+\cdots+a_{0} \in R[x]$ be a polynomial irreducible $\bmod m$ and $R_{0}=R[x] /(f)$. Then $R_{0}$ is a local separable $R$ algebra, free (and finitely generated) as an $R$-module, hence $R_{0}$ can be imbedded in a Galois extension $R_{0}^{\prime}$ of $R$ (see [8]). Moreover $R_{0}^{\prime}$ is a semilocal ring in which all residue fields contain at least $n$ elements. Since

$$
M \otimes_{R} R^{\prime} \otimes_{R} R_{0}^{\prime} \approx_{A} \otimes_{R^{\prime}} \otimes_{R_{0}} N \otimes_{R} R^{\prime} \otimes_{R} R_{0}^{\prime}
$$

hence it follows from the lemma that $M \otimes R_{0}^{\prime} \approx_{A} \otimes_{R_{0}} N \otimes R_{0}^{\prime}$. Therefore it suffices to prove the theorem in the case where $R^{\prime}$ is a Galois extension of $R$. In that case all $A$-isomorphism classes of $A$-modules $M^{\prime}$ such that $M^{\prime} \otimes R^{\prime} \approx{ }_{A} \otimes R^{\prime} M \otimes R^{\prime}$ can be considered as elements of the set $H^{1}\left(R^{\prime} / R\right.$, Aut $\left._{A} \otimes \ldots(M \otimes \cdots)\right)$. For any flat $R$-algebra $P$,

$$
\operatorname{Aut}_{A} \otimes_{P}(M \otimes P)=\left(\operatorname{End}_{A} \otimes_{P}(M \otimes P)\right)^{*}=\left(\operatorname{End}_{A}(M) \otimes P\right)^{*}
$$

(see (A) in $\$ 1$ ). We shall show that the $R$-algebra $\operatorname{End}_{A}(M)$ is of type (*). Let $\phi$ be the canonical homomorphism $\operatorname{End}_{A}(M) \rightarrow \operatorname{End}_{A}(M) \otimes R / m$ and let $z \in \operatorname{End}_{A}(M) \otimes R^{\prime}$. If $(\phi \otimes 1)(z)$ is invertible in $\operatorname{End}_{A}(M) \otimes R / m$ then the endomorphism of $M \otimes R / m \otimes R^{\prime}$ induced by $z$ is invertible. Thus it follows from Corollaire 1, p. 105 in [1] that $z$ is a surjective endomorphism of $M \otimes R^{\prime}$. Since $M \otimes R^{\prime}$ is a finitely presented $R^{\prime}$ module hence (by Proposition 8.9.3 of [6]) $z$ is an automorphism. Thus $z \in\left(\operatorname{End}_{A}(M) \otimes R^{\prime}\right)^{*}$.

Hence we may apply Theorem 1 . Thus the set

$$
H^{1}\left(R^{\prime} / R, \operatorname{Aut}_{A} \otimes \ldots(M \otimes \cdots)\right)
$$

is trivial and the theorem is proved.

The following result generalizes Corollary 76.9 in [4] to the case of any noetherian local ring.

COROLlARY 1. Let $R$ be a local noetherian ring, $m$ its maximal ideal, let $\hat{R}$ be the completion of $R$ in the $m$-adic topology and let $A$ be a finitely generated $R$-algebra. Then for any $A$-modules $M, N$, finitely presented over $R, M \otimes \hat{R} \approx \triangle_{\Lambda} \otimes_{R} N \otimes \hat{R}$ if and only if $M \approx{ }_{\Delta} N$. 
Proof. The result follows from Theorem 2 and the well-known theorem (see, e.g., [2, Proposition 9, p. 72]) asserting that $\hat{R}$ is faithfully flat over $R$.

The next corollary gives a generalization of theorems of NoetherDeuring [4, Theorem 29.7] and of Reiner-Zassenhaus [4, Theorem $76.20]$.

COROLLARY 2. Let $R$ be a valuation ring, let $K$ be the field of fractions of $R$ and let $A$ be a finitely generated $R$-algebra. Let $L$ be a field containing $K$ as a subfield and finite dimensional over $K$. Let $R^{\prime}$ be any local subring of $L$ which dominates $R$ and has $L$ as its field of fractions. Then for any $A$-modules $M, N$, finitely presented as $R$-modules, $M \otimes R^{\prime}$ $\approx A \otimes R^{\prime} N \otimes R^{\prime}$, if and only if $M \approx_{A} N$.

Proof. Since $R$ is a valuation ring hence any $R$-module without torsion is flat. Hence $R^{\prime}$ is a flat $R$-module, since $R^{\prime}$ dominates $R$ it is in fact a faithfully flat $R$-module. Thus the corollary follows from Theorem 2.

It follows from the Remark on p. 375 that Theorem 2 can be strengthened to the case where $R$ is a semilocal ring. This stronger result gives a generalization of Proposition 2.5.8 (b) of [6]. Moreover part (a) of the proposition follows easily from the above result.

\section{REFERENCES}

1. N. Bourbaki, Algèbre commutative, Chapitres 1, 2, Actualités Sci. Indust., no. 1290, Hermann, Paris, 1961. MR 36 \#146.

2. - Algèbre commutative, Chapitres 3, 4, Actualités Sci. Indust., no. 1293, Hermann, Paris, 1961. MR 30 \#2027.

3. S. U. Chase, D. K. Harrison and A. Rosenberg, Galois theory and Galois cohomology of commutative rings, Mem. Amer. Math. Soc. No. 52 (1964), 15-33. MR 33 \#4118.

4. Ch. W. Curtis and I. Reiner, Representation theory of finite groups and associative algebras, Pure and Appl. Math., vol. XI, Interscience, New York, 1962. MR 26 \#2519.

5. A. Grothendieck, Technique de descente et theorèmes d'existence en geometrie algébrique. I: Généralités. Descente par morphismes fidèlement plats, Séminaire Bourbaki, 1959/60, Exposé 190, Secrétariat mathématique, Paris, 1960. MR 23 \#A2273.

6. - Eléments de gêométrie algébrique, Inst. Hautes Etudes Sci. Publ. Math. Nos. 20, 24, 28 (1964/65). MR 30 \#3885; MR 33 \#7330; MR 36 \#178.

7. J. P. Serre, Corps locaux, Publ. Inst. Math. Univ. Nancago, VIII, Actualités Sci. Indust., no. 1296, Hermann, Paris, 1962. MR 27 \#133.

8. O. E. Villamayor, Separable algebras and Galois extensions, Osaka J. Math. 4(1967), 161-171. MR 37 \#064.

Institute of Mathematics, Polish Academy of Sciences, Warsaw, Poland 